\title{
The impact of post PCNL tube type on blood loss and postoperative pain
}

\author{
Salman Jamil ${ }^{1}$, M. Hammad Ather $^{2}$
}

\begin{abstract}
Objective: To evaluate the impact of nephrostomy tube type on postoperative pain and blood loss following percutaneous nephrolithotomy (PCNL).

Methods: This is a prospective non-randomized study performed at Aga Khan University Hospital from July 2017 to June 2018. In this study we prospectively studied adult patients (16 to 65 years) who underwent unilateral PCNL. Patients who had nephrostomy with balloon (12Fr Foley's catheter) were compared with patients who had nephrostomy without balloon (12Fr Nelaton ${ }^{T M}$ catheter). STONE Nephrolithometry score was used to assess the stone complexity. Mean pain score at six and 24 hours and mean hemoglobin drop at 24 hours was compared between two groups using independent sample t-test, $p$-value of $<0.05$ was considered significant.

Results: Over one year, 198 PCNL were performed out of which 119 were included for analysis. Sixty-six had nephrostomy tube with balloon and 53 had nephrostomy tube without balloon. Mean STONE score $(9.66 \pm 1.4$ vs. $9.64 \pm 1.24)$ and operative time $(72.84 \pm 28.34$ vs. $86.05 \pm 32.1$ minutes $)$ was comparable. Mean postoperative pain score at 6 hours and 24 hours postoperative was significantly lower in balloon group as compared to without balloon group. Mean Hemoglobin drop was similar in both groups $(p=0.60)$.

Conclusion: The use of nephrostomy tube with balloon after PCNL as this is associated with less pain and comparable hemoglobin drop as compare to nephrostomy tube without balloon.
\end{abstract}

KEYWORDS: Percutaneous nephrostomy (PCN), Percutaneous Nephrolithotomy (PCNL), Perc, Pain.

doi: https://doi.org/10.12669/pjms.36.3.1558

How to cite this:

Jamil S, Ather MH. The impact of post PCNL tube type on blood loss and postoperative pain. Pak J Med Sci. 2020;36(3):402-406. doi: https://doi.org/10.12669/pjms.36.3.1558

This is an Open Access article distributed under the terms of the Creative Commons Attribution License (http://creativecommons.org/licenses/by/3.0), which permits unrestricted use, distribution, and reproduction in any medium, provided the original work is properly cited.

\section{INTRODUCTION}

1. Salman Jamil,

2. M. Hammad Ather,

1,2: Department of Surgery (Urology),

Aga Khan University,

Karachi, Pakistan.

Correspondence

M. Hammad Ather, FCPS (Urol), FRCS, FEBU.

Professor and Head of Department of Surgery (Urology),

Aga Khan University Hospital,

P.O. Box 3500, Stadium Road,

Karachi-74800, Pakistan.

E-mail: hammad.ather@aku.edu

* Received for Publication:

August 24, 2019

* Revision Received:

* Revision Accepted:
November 18, 2019
December 5, 2019
PCNL is the standard of care for patients with moderate to large upper tract urinary stones. Despite being minimally invasive it can cause significant pain and sometimes lead to lifethreatening complications such as bleeding, sepsis and peri-renal visceral injury. ${ }^{1}$ Multiple factors influence postoperative pain and haemoglobin drop. Most of them e.g. body mass index, stone size and stone location are patient and stone related and cannot be modified. ${ }^{2}$ However there are factors which can be modified to reduce postoperative pain and hemoglobin drop e.g. ultrasound-guided access, use of Amplatz ${ }^{\mathrm{TM}}$ or balloon dilatation systems, reducing the operative 
time, staging the procedure in cases of a large stone burden and placement of a nephrostomy tube. ${ }^{3}$

Nephrostomy tube type is a modifiable factor, which can potentially influence postoperative pain and haemoglobin drop. Su H et al. in $2015^{4}$ compared the effect of nephrostomy tubes with and without balloon after percutaneous nephrolithotomy. They observed significantly less hemoglobin drop in nephrostomy tube with balloon as compared to those without balloon at 72 hours postoperatively, however noted no difference in postoperative pain. Jiang and colleagues ${ }^{5}$ however noted that the drainage types after PCNL using a nephrostomy tube, a double J stent or an openended ureteral catheter were equally safe and efficacious. They also observed that compared to a nephrostomy tube or an open-ended ureteral catheter double J stent adversely affect HRQoL. In a systematic review of recent publications Lee et al. ${ }^{6}$ noted that for hemoglobin changes, total tubeless and finer caliber percutaneous procedure are better than other methods. However, in order to improve hospital stay, total tubeless and tubeless PCNLs with stent may be superior to other procedures. In the current work we have attempted to assess the impact of type of nephrostomy placed post PCNL have influence on postoperative pain and hemoglobin drop.

\section{METHODS}

This is a prospective non-randomized study performed at Aga Khan University Hospital from July 2017 to June 2018. Adult patients 16 to 65 years of age undergoing unilateral PCNL with single tract using 26Fr Amplatz ${ }^{\mathrm{TM}}$ sheath for stones in kidney confirmed on non-contrast CT abdomen were included. Patients who previously had open renal surgery on the same side or had a percutaneous nephrostomy already in place or raised serum creatinine $(>1.2 \mathrm{mg} / \mathrm{dL})$, raised INR $(>1.3)$ or reduced platelet $\left(<100 / \mathrm{mm}^{3}\right)$ were excluded.

After institutional review committee approval (Ref: 4649-SUR-ERC-17 dated May 3, 2017), all patients who fulfill the inclusion criteria were included in the study following an informed consent. Consultant surgeon with more than five years of experience performed PCNL. Procedure was done in prone position after placing ureteric catheter in the same general anesthesia using a flexible cystoscope in supine position. Tract was dilated using serial metallic dilators. Stone fragmented with ultrasonic probe using EMSTM lithoclastmaster. At the end of procedurea $12 \mathrm{Ch}$., nephrostomy tube, both with or without balloon, was placed and clamped overnight. Patients who had $12 \mathrm{Ch}$. Foley's catheter, with balloon inflated (approx. 1-1.5 cc) placed as nephrostomy were included in Group-A. Patients with 12Ch. Nelaton catheter ${ }^{\mathrm{TM}}$ placed as nephrostomy were included in Group-B. Choice of nephrostomy was left on operating surgeon's discretion. Nephrostomy was de-clamped on first post-operative day and subsequently removed on $2-5^{\text {th }}$ postoperative day at the discretion of the admitting surgeon. Post-operative hemoglobin and hematocrit were measured at 24 hours. All patients received paracetamol 1g 6hrly and Tramadol 50mg 8hrly for postoperative pain. Pain was assessed at 6 hours and 24 hours post-operatively.

Data was analyzed through SPSSTM version 21 . Mean and standard deviation was calculated for quantitative variables i.e. age, BMI, stone size, density (HU unit) and pain score on VAS. Frequency

Table-I: Baseline parameters.

\begin{tabular}{|c|c|c|c|}
\hline & $\begin{array}{l}\text { Group-A } \\
\text { (Foley's } \\
\text { catheter) }\end{array}$ & $\begin{array}{l}\text { Group-B } \\
\text { (Nelaton } \\
\text { catheter) }\end{array}$ & p-value \\
\hline TOTAL (n) & 66 & 53 & \\
\hline Age (years) & $44.47+/-13.6$ & $44.94+/-14.5$ & 0.181 \\
\hline Female & 18 & 16 & \\
\hline Male & 48 & 37 & \\
\hline BMI & $26.2+/-4.2$ & $26.8+/-3.9$ & 0.418 \\
\hline Stone size & $22.27+/-8.03$ & $20.68+/-6.79$ & 0.246 \\
\hline Mean HU & $1065.9+/-298.33$ & $1019+/-329.94$ & 0.428 \\
\hline Tract length & $85.96+/-23.8$ & $92.0+/-25.25$ & 0.183 \\
\hline Operative time & $72.84+/-28.34$ & $86.05+/-32.1$ & 0.021 \\
\hline \multicolumn{4}{|c|}{ Hydronephrosis } \\
\hline GRADE 0 & 9 & 11 & \\
\hline GRADE 1 & 12 & 3 & \\
\hline GRADE 2 & 22 & 30 & \\
\hline GRADE 3 & 18 & 5 & \\
\hline GRADE 4 & 5 & 4 & \\
\hline \multicolumn{4}{|c|}{ No. of calyces involved } \\
\hline 1 & 12 & 5 & \\
\hline 2 & 26 & 37 & \\
\hline 3 & 16 & 7 & \\
\hline 4 & 12 & 4 & \\
\hline STONE Score & $9.66+/-1.44$ & $9.64+/-1.24$ & 0.935 \\
\hline $\begin{array}{l}\text { Mean pre op } \\
\text { Hemoglobin. }\end{array}$ & $13.48+/-2.27$ & $13.64+/-1.90$ & 0.673 \\
\hline $\begin{array}{l}\text { Mean } 24 \text { hrs. } \\
\text { Hemoglobin. }\end{array}$ & $12.40+/-2.18$ & $12.30+/-2.35$ & 0.816 \\
\hline Mean pre op Hct. & . $41.01+/-5.88$ & $41.59+/-5.22$ & 0.565 \\
\hline Mean 24 hrs. Hct. & $37.03+/-7.00$ & $38.23+/-5.63$ & 0.303 \\
\hline
\end{tabular}


Salman Jamil et al.

Table-II: Comparison of outcome parameters.

\begin{tabular}{lccc}
\hline & Group-A & Group-B & p-value \\
\hline Mean Pain Score 6HRS & $4.76+/-0.81$ & $5.24+/-0.97$ & 0.004 \\
Mean Pain Score 24HRS & $2.26+/-0.91$ & $2.69+/-1.06$ & 0.020 \\
Mean Hemoglobin drop & $1.08+/-0.7$ & $1.14+/-0.69$ & 0.60 \\
Meant Hct drop & $3.43+/-2.21$ & $3.41+/-1.97$ & 0.97 \\
\hline
\end{tabular}

Table-III: Comparison of outcome parameters.

\begin{tabular}{|c|c|c|c|c|c|}
\hline & & Group-A & Group-B & Total & p-value \\
\hline \multirow[t]{2}{*}{ Complete Clearance } & Yes & 55 & 47 & 102 & 0.41 \\
\hline & No & 11 & 6 & 17 & \\
\hline \multirow[t]{2}{*}{ JJ Stenting } & Yes & 36 & 29 & 65 & 0.98 \\
\hline & No & 30 & 24 & 54 & \\
\hline \multirow[t]{2}{*}{ No. of Transfusions } & Yes & 3 & 1 & 4 & 0.26 \\
\hline & No & 63 & 52 & 114 & \\
\hline
\end{tabular}

and percentages were calculated for qualitative variables, i.e. gender, hydronephrosis. Mean postoperative hemoglobin drop was calculated after subtracting $24 \mathrm{hr}$ post op hemoglobin from preoperative hemoglobin. of each patient. Mean pain score was calculated at 6 and $24 \mathrm{hrs}$. Mean postoperative pain and hemoglobin drop of 2 groups were compared using independent sample student t-test. Effect modifiers were controlled through stratification of age, sex, and stone size and STONE score. ${ }^{7}$

\section{RESULTS}

A total of 198 cases had this procedure during the study period out of which 119 were included for final analysis, 66 were in Group-A and 53 in Group-B . Baseline parameters are detailed in Table-I. Both groups were comparable in terms of age, BMI and stone complexity. Complete clearance was achieved in 102 patients and JJ stent was placed in 65 patients. Only four patients required transfusion, none of the patient required angioembolization.

There was no significant difference in postoperative Hemoglobin drop between two groups, however mean postoperative pain score at six hours and 24 hours was signifcanlty lower in Group-A (Tables-II and III). Multivariate regression analysis showed that expected mean pain scores at 6 hours is 0.474 units more among those without balloon as compared to those with balloon after adjusting for other covariates. BMI, JJ stenting and Nephrostomy tube were found to be significant independent predictors of pain at 24hours. The expected mean pain scores after 24 hours is 0.389 units more among those without balloon as compared to those with balloon after adjusting for other covariates (Table-IV).

\section{DISCUSSION}

Percutaneous nephrolithotomy (PCNL) despite being minimally invasive can cause significant pain and blood loss. ${ }^{8}$ Post operative pain is the most common concern in immediate postoperative period. There are many ways of reducing postoperative pain. These include local anesthetic infiltration into the tract ${ }^{9}$ reducing the renal pelvic

Table-IV: Multivariate analysis of outcome parameters.

\begin{tabular}{lcccc}
\hline \multicolumn{5}{c}{ Pain at 6 hours } \\
\hline & Odds Ratio & Std. Error & p-value & $95.0 \%$ Confidence Interval \\
\hline (Constant) & 3.957 & 0.340 & 0.000 & $3.283-4.631$ \\
Nephrostomy Tube & 0.474 & 0.165 & 0.005 & $0.148-0.801$ \\
Gender & 0.261 & 0.181 & 0.151 & $-0.097-0.619$ \\
\hline \multicolumn{4}{c}{ Pain at 24 hours } \\
\hline (Constant) & 0.625 & 0.734 & $-1.451-1.025$ \\
Nephrostomy Tube & -0.213 & 0.173 & 0.026 & $0.047-0.731$ \\
BMI & 0.389 & 0.021 & 0.001 & $0.029-0.113$ \\
JJ Stenting & 0.071 & 0.175 & 0.005 & $0.149-0.840$ \\
\hline
\end{tabular}




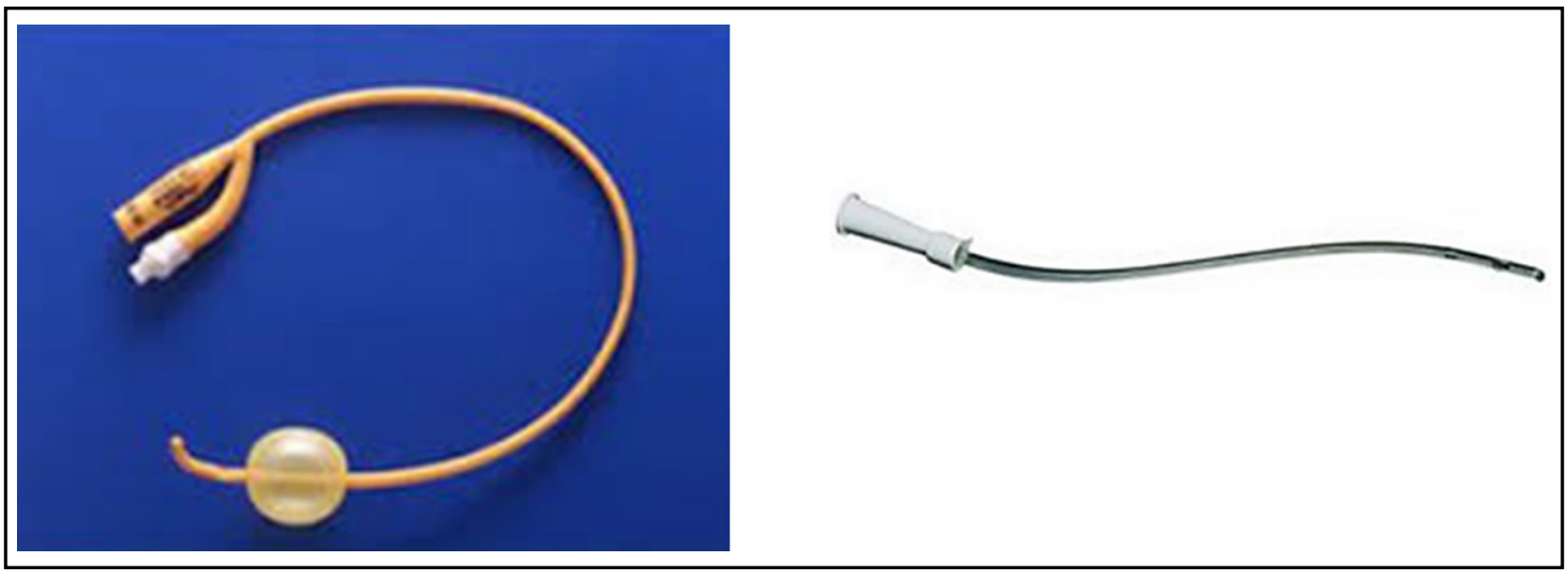

Fig.1: Nephrostomy tube types. 1a: With balloon, 1b: without balloon.

pressure during surgery, paravertebral block (T11-L1), using postoperative opioid analgesia, reducing the size of nephrostomy tube or not placing a nephrostomy tube at all..$^{10-13}$

Multiple studies have shown that tubeless PCNL is safe and feasible for select group of patients. However, keeping nephrostomy at end of procedure is desirable in some conditions e.g. residual stone, longer operative time, more than 1 percutaneous access, significant perforation of the collecting system and significant bleeding. ${ }^{14}$ It is well established that decreasing the size of nephrostomy tube can decrease post-operative pain. ${ }^{15}$ However, the effect of nephrostomy tube type on pain is less well studied. We measured pain at six and 24 hours post-operatively and found significantly less pain in balloon nephrostomy group, which may be attributable to softer latex material of balloon tube. In the current work we noted a softer balloon catheter (Foleys 12 Fr.) is much better tolerated compared to a firmer tube (Nelaton ${ }^{\mathrm{TM}}$ catherther12 Fr.) Fig.1 $a$ and $b$.

Postoperative bleeding is another major concern following PCNL. This bleeding is either parenchymal or arterial. Significant delayed bleeding is almost always due to pseudoaneurysms or arteriovenous fistulae. Serious arterial injury requiring angioembolization is around $1 \%$ in different studies. ${ }^{16}$ Parenchymal bleeding can be controlled by advancing working sheath during the procedure. However, the tamponade is lost at the end of procedure. This tamponade effect is provided postoperatively by a Foley catheter with a balloon inflated. Arterial bleeding is relatively rare and usually from a tiny arteriole and may occurs during puncture or dilatation of a tract.
This type of bleeding can de dealt postoperatively by providing tamponade. Nephrostomy tube with balloon is potentially better for providing this tamponade as shown by $\mathrm{Su}$ et al. ${ }^{4}$ they compared nephrostomy tube with balloon and without balloon but found no significant difference in postoperative pain. In their study the primary endpoint was hemoglobin drop and they applied traction over nephrostomy, which can affect pain. Our results showed no differnece in hemoglobin drop between two groups perhaps as we did not keep nephrostomy tube on traction postoperatively that may affect hemoglobin drop.

Limitations of the study: This was an interventional study however randomization was not done. Nephrostomy tube with balloon was used by single surgeon whereas Nelaton catheter was used by others, this may have contributed to differences in postoperative pain and operative time. Whether to clamp nephrostomy before removal and when to remove ureteric and urethral catheter was left on discretion of operating surgeon.

\section{CONCLUSION}

The present study supports the use of nephrostomy tube with balloon (Foley's catheter) after PCNL as this is associated with less pain and comparable hemoglobin drop as compare to nephrostomy tube without balloon (Nelaton catheter). Further randomized controlled trails are needed to compare both the tubes.

Conflict of interest: None.

Funding: None. 


\section{REFERENCES}

1. Un S, Cakir V, Kara C, Turk H, Kose O, Balli O, et al. Risk factors for hemorrhage requiring embolization after percutaneous nephrolithotomy. Can Urol Assoc J. 2015;9(9-10):E594-E598. doi: 10.5489/cuaj.2803

2. Lee JK, Kim BS, Park YK. Predictive factors for bleeding during percutaneous nephrolithotomy. Korean J Urol 2013;54(7):448-453. doi: 10.4111/kju.2013.54.7.448

3. Akman T, Binbay M, Sari E, Yuruk E, Tepeler A, Akcay $\mathrm{M}$, et al. Factors affecting bleeding during percutaneous nephrolithotomy: single surgeon experience. J Endourol. 2011;25(2):327-333. doi: 10.1089/end.2010.0302

4. Su H, Zhu Y, Wang J, Deng Q, Pei L, Wang J. Application of nephrostomy tubes with balloon after percutaneous nephrolithotomy: A randomized controlled clinical trial. Int J Urol. 2015;22(12):1118-1121. doi: 10.1111/iju.12942

5. Jiang H, Huang D, Yao S, Liu S. Improving Drainage After Percutaneous Nephrolithotomy Based on HealthRelated Quality of Life: A Prospective Randomized Study. J Endourol. 2017;31(11):1131-1138. doi: 10.1089/ end.2017.0444

6. Lee JY, Jeh SU, Kim MD, Kang DH, Kwon JK, Ham WS, et al. Intraoperative and postoperative feasibility and safety of total tubeless, tubeless, small-bore tube, and standard percutaneous nephrolithotomy: A systematic review and network meta-analysis of 16 randomized controlled trials. BMC Urol. 2017;17(1):48. doi: 10.1186/s12894-017-0239-x

7. Molina WR, Kim FJ, Spendlove J, Pompeo AS, Sillau S, Sehrt DE. The STONE Score: a new assessment tool to predict stone free rates in ureteroscopy from pre-operative radiological features. Int Braz J Urol. 2014;40(1):23-29. doi: 10.1590/S1677-5538.IBJU.2014.01.04

8. Zhu W, Liu Y, Liu L, Lei M, Yuan J, Wan SP, et al. Minimally invasive versus standard percutaneous nephrolithotomy: A meta-analysis. Urolithiasis. 2015;43(6):563-570. doi: $10.1007 /$ s00240-015-0808-y

9. Hosseini SR, Imani F, Shayanpour G, Khajavi MR. The effect of nephrostomy tract infiltration of ketamine on postoperative pain and peak expiratory flow rate in patients undergoing tubeless percutaneous nephrolithotomy: A prospective randomized clinical trial. Urolithiasis. 2017;45(6):591-595. doi: 10.1007/s00240-017-0971-4
10. Alsyouf M, Abourbih S, West B, Hodgson H, Baldwin DD. Elevated renal pelvic pressures during percutaneous nephrolithotomy risk higher postoperative pain and longer hospital stay. J Urol. 2018;199(1):193-199. doi: 10.1016/j. juro.2017.08.039

11. Lojanapiwat B, Chureemas T, Kittirattarakarn P. The efficacy of peritubal analgesic infiltration in postoperative pain following percutaneous nephrolithotomy-A prospective randomized controlled study. Int Braz J Urol. 2015;41(5):945952. doi: 10.1590/S1677-5538

12. Arshad Z, Zaidi SZ, Jamshaid A, Qureshi AH. Post operative pain control in percutaneous nephrolithotomy. J Pak Med Assoc. 2018;68(5):702-704.

13. Hatipoglu Z, Gulec E, Turktan M, Izol V, Arıdogan A, Gunes Y, et al. Comparative study of ultrasoundguided paravertebral block versus intravenous tramadol for postoperative pain control in percutaneous nephrolithotomy. BMC Anesthesiol. 2018;18(1):24. doi: 10.1186/s12871-018-0479-7

14. Limb J, Bellman GC. Tubeless percutaneous renal surgery: review of first 112 patients. Urology. 2002;59(4):527-531. doi: 10.1016/s0090-4295(01)01627-2

15. PietrowPK,AugeBK,Lallas CD,Santa-CruzRW,NewmanGE, Albala DM, et al. Pain after percutaneous nephrolithotomy: Impact of nephrostomy tube size. J Endourol. 2003;17(6):411414. doi: $10.1089 / 089277903767923218$

16. Keoghane SR, Cetti RJ, Rogers AE, Walmsley BH. Blood transfusion, embolisation and nephrectomy after percutaneous nephrolithotomy (PCNL). BJU Int. 2013;111(4):628-632. doi: 10.1111/j.1464-410X.2012.11394.x

\section{Authors' Contribution:}

SJ and MHA: Protocol/project development, Data collection or management, Data analysis, Manuscript writing/editing, are responsible for integrity of research. 